\title{
AGENT-BASED SUPPLY CHAIN PLANNING IN THE FOREST PRODUCTS INDUSTRY
}

\author{
Sophie D'Amours' ${ }^{1,2}$, Jean-Marc Frayret', Alain Rousseau', \\ Steve Harvey ${ }^{1}$, Pascale Plamondon ${ }^{1}$, Pascal Forget ${ }^{1}$ \\ ${ }^{\prime}$ Research Consortium in e-Business in the Forest Products Industry (FOR@C) \\ ${ }^{2}$ Network Organization Technology Research Center (CENTOR) \\ Université Laval, Quebec, Canada \\ Sophie.Damours@forac.ulaval.ca
}

\begin{abstract}
Due to new economical challenges and recent trends regarding international trade and globalization, many companies from the Canadian forest products industry are now facing the need to reengineer their organizational processes and business practices with their partners. This paper proposes an architectwe which aims to enable the development of advanced planning systems for the forest products industry. This architecture combines agent technology with operational research, in order to take advantage of the ability of agent-based technology to integrate distributed decision problems, and the ability of operation research to solve complex decision problems This paper describes how this architecture has been configured into an advanced planning and scheduling tool for the lumber industry, and how it is being validated.
\end{abstract}

\section{INTRODUCTION}

Due to new economical challenges and recent trends regarding international trade and globalization, many companies from the Canadian forest products industry have reached the point where profit improvement cannot be reaped without the involvement of their entire organization (e.g., their distributed facilities and offices spread across North America and the world) as well as their business partners (e.g., raw material suppliers, industrial clients and distributors). If companies from other sectors have faced these challenges a few years ago (e.g., the semi-conductor and automotive industries), companies of the Canadian forest products industry are now facing the need to reengineer their organizational processes and business practices with their partners and adopt supply chain management best practices.

Supply chains are global networks of organizations where material and information flow in many directions within and across organizational boundaries through complex business networks of suppliers, manufacturers, and distributors, to the final customers. Organizations of the supply chain cooperate to improve the flows of materials and information in order to maximize customer satisfaction at the lowest possible cost and highest possible speed.

Please use the following format when citing this chapter:

D'Amours, S., Frayret, J.-M., Rousseau, A., Harvey, S., Plamondon, P., Forget, P., 2006, in IFIP International Federation for Information Processing, Volume 220, Information Technology for Balanced Manufacturing Systems, ed. Shen, W., (Boston: Springer), pp. 17-26. 
In that regard, the forest product supply chain is similar to other industries: forest product material flow from forest contractors, to lumber or paper or panel facilities, to value-added mills (referred to as secondary transformation), and through many channels of distributors and wholesalers to finally reach the markets. However, unlike the traditional manufacturing supply chain, which has a convergent product structure (i.e., assembly), the forest product industry needs to master industry specific production processes due to its divergent product structure (i.e., trees are broken down into many products) and the highly heterogeneous nature of its raw material, which make planning and control a difficult task. Because of this inherent complexity, the forest products industry needs to compensate its lack of control over these various stochastic elements by the ability to (1) exchange promptly information throughout its supply chain regarding supply availability and quality, production output and demand, and (2) quickly react in a coordinated manner with supply chain members to correct any deviances or disturbances to the plan. Here lies a need for reactive and specific information and decision support systems to address both the need to produce feasible operation plans and to quickly adapt these plans when perturbing events occur. The experimental planning platform presented in this paper attempts to address these two issues. In particular, an agent-based architecture is proposed in order to develop an experimental environment to design and test various configurations of planning systems for the forest products industry. The remaining of this paper is organized as follows. First, a review of the literature dealing with Advanced Planning and Scheduling systems (i.e., APS) and with agentbased manufacturing and supply chain management systems is presented. Next, the different elements of the proposed generic architecture are introduced. Then, the functional and technological aspects of a specific implementation of the proposed approached are illustrated. Finally, the validation process for correction and improvements is detailed.

\section{LITERATURE REVIEW}

Operation planning within supply chains is a complex issue. Companies usually face this by implementing and using information and decision support systems, which address various planning tasks. Some companies also adopt just-in-time approaches control the pace of production and replenishment using Kanban. This paper focuses on computer supported planning systems in a distributed planning environment.

\subsection{Advanced Planning and Scheduling}

Advanced Planning and Scheduling (APS) systems are considered by many as the state of the art of manufacturing and supply chain planning and scheduling practices. The reader is referred to Stadtler and Kilger (2000) and Stadtler (2005) for a thorough description of APS. These systems usually exploit operations research (OR) technologies or constraint programming in order to carry out in an integrated manner true finite capacity planning and scheduling optimization at the long, mid and short term levels of decision making (Fleischmann and Meyr (2003)). For a specific description of some of the many planning problems and optimization applications in the forest product industry, the reader is referred to Rönnqvist 
(2003), Epstein, Morales, Seron, and Weintraub (1999) and Frayret, Boston, D'Amours, and Lebel (2005).

\subsection{Agent-based manufacturing and supply chain management systems}

In order to address the planning and scheduling of manufacturing and supply chain systems, academics have initiated in the middle of the 1980s a new body of approaches and distributed computing techniques drifting away from traditional ORbased solutions. Some of these approaches are referred to as agent-based manufacturing and supply chain management systems. They are designed to tackle the need for reactive, reliable, and (re)configurable operation management systems. These approaches are rooted in the multi-agent technologies which are related to distributed artificial intelligence (Weiss (1999)).

An agent-based manufacturing system may be defined as a planning and control system made of interdependent software agents designed to (1) individually handle a part of a manufacturing planning and control problem, such as planning a single order or allocating tasks to resources, and (2) collectively carry out specific higher functionalities such as planning an entire manufacturing system. Software agents generally exhibit characteristics that allow them to individually behave and interact with each other in such a manner that they collectively fulfill the purpose of the entire system. In their book, Shen, Norrie, and Barthes (2001) identify 12 desirable characteristics of an agent: network-centric, communicative, semi-autonomous, reactive, deliberative, collaborative, pro-active, predictive, adaptive, flexible, persistent, mobile.

Many agent-based approaches for the planning and control of supply chains and manufacturing activities have been proposed in the literature. For a review of these approaches, the reader is referred to Shen and Norrie (1999) and Parunak (1999). The reader is also referred to Caridi and Cavalieri (2004) who provide a recent critical analysis of multi-agent technology applied to manufacturing. Their analysis notably reveals the lack of real world applications and the low maturity level of agent-based manufacturing technology. The reader is referred to Tharumarajah (2001) and Frayret, D'Amours, and Montreuil (2004) who analyze some of the important issues related to the design of such distributed systems, including the distribution of decisions and the coordination of manufacturing activities. These recent developments provide many new concepts and ideas to design solutions to support interdependent supply chain members to make their planning and control decisions so as to be collectively more efficient than if they were not coordinated.

\section{GENERIC ARCHITECTURE}

The architecture that is introduced in this paper aims at addressing two particular issues relevant to provide the forest products industry with specific normative decision and planning tools. The first issue concerns the ability to plan and coordinate operations throughout the supply chain, while the second issue concerns the ability to analyze through simulation the dynamics and performance of various supply chain scenarios. In order to address these challenges, an experimental platform was developed by the FOR@C Research Consortium, which is a joint 
industry and university research initiative focused on supply chain planning within the forest product industry. The design of this platform exploits both multi-agent technology and OR techniques. Figure 1 presents the different functions of this platform.

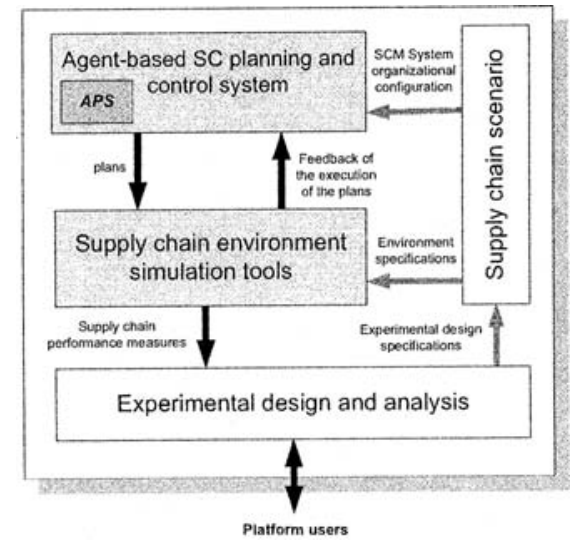

Figure 1 - General overview of the experimental platform

\subsection{Architecture description}

In the context of this paper, the architecture is defined through its generic principles, mechanisms and software components used to build particular implementations of agent-based supply chain planning systems. The description of this architecture can be made from three different perspectives: the business level, the planning level and the implementation level.

The business level of the architecture encompasses the organizational principles that are used to design a supply chain business configuration from which a detailed configuration of an agent-based supply chain planning system is based on. These principles, inspired by previous work (Montreuil, Frayret, and D' Amours (2000), Frayret (2002)), propose to model supply chains as networks of autonomous and interdependent business units responsible for fulfilling the agreements and commitments they have with their partners. From a company's point of view, a business unit can also be external entities that represent a business partner such as a customer, a supplier or a contractor.

The planning level of the architecture consists in all the generic structures and mechanisms required to implement agent-based supply chain planning systems for the forest products industry. First, there is a direct structural relationship between a business units' supply chain configuration and its corresponding distributed planning configuration. In other words, there is a direct correspondence between business units and their planning level counterpart, referred to as a Planning Unit (PU). Consequently, PUs' relationships are specified through a supply chain business configuration. Because of the flexibility of the business level principles, the scope of a PU can vary greatly. For example, a PU can be set to support the operation planning of a department, a complete facility, or an entire multi-facility division. Second, the internal configuration of a PU is composed of specialized agents capable 
of maintaining complex communication and collaborative workflows with each other (whether they are in the same PU or not) through structured conversations based on specifically designed conversation protocols, commonly used in multiagent systems. These configurations are built and customized in order to implement the particular planning support models of their corresponding PU's decision processes. Figure 2 presents a specific implementation of a supply chain configuration, including different PUs.

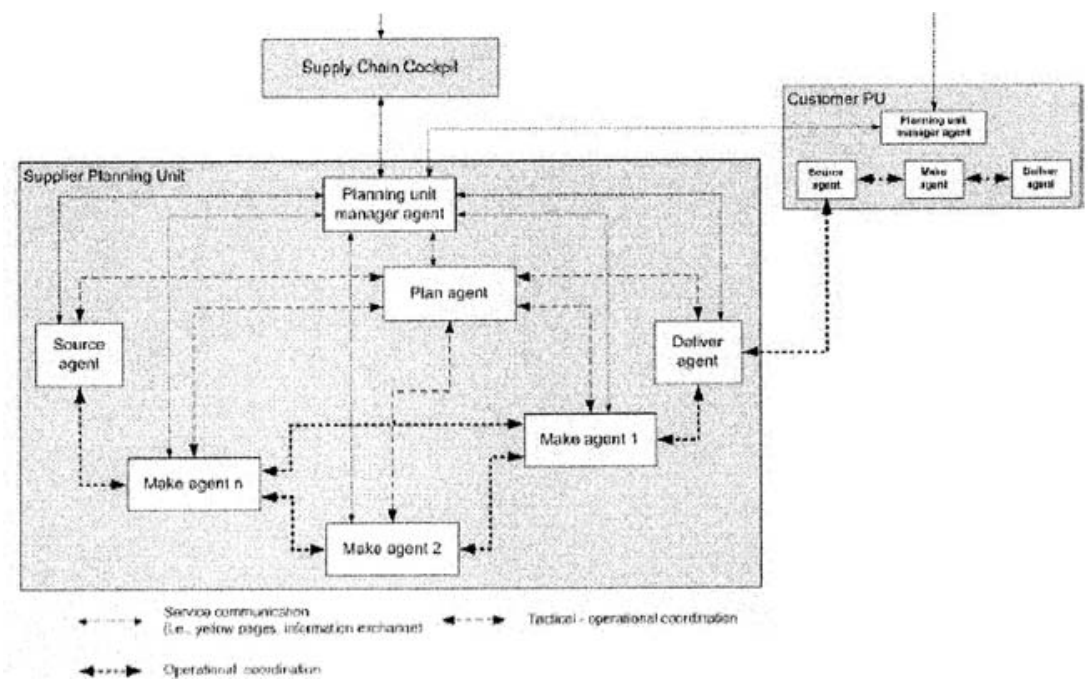

Figure 2 - Components of a specific implementation of a supply chain configuration

The configuration of an agent-based supply chain planning system specifies the PU's internal configurations and its relationships with other PUs. Its design and implementation, which includes the customization of the PU, is made through a software component called Supply Chain Modeler. Supply Chain Modeler enables system designers to customize and deploy PUs through a network of computer.

\subsection{Planning unit configuration}

Within a PU, planning activities are carried out by various agents responsible for the many planning functions of the planning process model. This functional distribution is inspired by the SCOR model defined by the Supply Chain Council (Stephens (2000)) and the agent-based supply chain management application presented in Fox, Barbuceanu, and Teigen (2000). Each PU has a deliver agent which function is to manage all relationships with the PU's customers. On the other side of the functional spectrum, each PU has also a source agent which role is to manage relationships with all the PU's suppliers. One function of the Source Agent is to forward to the right suppliers the needs of the PU that cannot be fulfilled internally or for which it is more economical to outsource the fulfillment. If the PU is responsible for carrying out production activities (i.e., it is not a warehouse or a distribution center), it is geared up with a set of make agents. According to the 
planning process model, production planning functions can be assigned to one or many make agents responsible for a part of the overall planning functions. This way, the planning functions can be distributed and assigned to make agents responsible for the planning of production cells which manufacture of a product family. The flexibility of this decomposition approach is necessary for the design of specific decision support tools that are specialized and adapted to the production process, as opposed to a generic one.

All agents are responsible for continuously monitoring their own environment and react to certain changes that may occur. Also, because agents interact with each other, their environment is also made of all messages received from other agents specifying a new or modified requirement plan, a new or modified replenishment plan, a contingency situation, or a high priority requirement to process. Although most agents in the reviewed literature are mainly reactive (i.e., they are designed to carry out specific tasks triggered when specific events occur), the agents envisioned in the proposed approach can also exhibit a pro-active behavior by not only reacting to changes in their environment, but also by initiating actions that could improve their performance. In this context, it appears that the notion of agent goals is fundamental as it guides the agents' decision-making behavior toward the achievement of their goals. The planning process model of an agent explicitly describes such objectives.

\subsection{Collaborative environment}

Beyond the description of the planning process, one of the main challenges of designing an agent-based supply chain planning system is to create a collaborative environment that allows each agent geared up with advanced planning tools to share and exchange in time partial or factual decisions, or constraints in order to make sure that the final planning decisions are made jointly with respect to the agents' constraints and their collective performance. As mentioned previously, Dudek and Stadtler (2005) propose such an approach and describe it from a planning process modeling perspective. From an implementation perspective, each agent must be configured so as to behave according to the collaborative planning process model, all the while being able to fulfill its local tasks and interact with local users.

In order to address both technological and planning process integration challenges, we rely on the concepts of conversation protocols that are commonly used in multi-agent systems. These protocols are used to specify how agents should collaborate through structured exchange of messages. Agents' collaborative behavior is then defined through internal workflows associated to each potential states of a conversation. At each of these states, each agent participating in the conversation must perform tasks to continue or stop the conversation. In our experimental planning platform, each agent has the ability to receive and send messages. Because planning the operations of a part of a supply chain in collaboration with other agents requires the ability to cope with various situations that cannot all be identified in advanced (i.e., at the configuration stage of the system), it may be necessary to use more advanced agents that can identify themselves which tasks to perform when facing a new situation. From an implementation perspective, conversation protocols are inspired from the FIPAACL3 standards for the interoperation of heterogeneous software agents. 


\section{APPLICATION TO THE LUMBER PRODUCTION PLANNING PROBLEM}

The generic architecture presented in the previous section has been configured and specific operations planning agents have been designed in order to carry out the planning and coordination of the lumber production and distribution. The next sections introduce this particular application.

\subsection{Introduction to the lumber supply chain}

The lumber supply chain that is introduced here is a part of the entire forest product supply chain. In particular, it represents the lumber production and distribution part of it, also referred to as the first transformation. In brief, as it is usually the case in Québec, Canada, forest is harvested by small size entrepreneurs responsible for felling trees and for crosscutting them into appropriate length logs. Once in the mills, logs remain in the log yard until there are broken down into various sizes of rough pieces of lumber. A sawing process represents the use of a particular sawing pattern on a particular log class. The concept of sawing process allows the control the co-production predictability through the definition of more or less detailed classes of logs. Next, bundles of same dimension pieces of lumber (e.g., $2 \times 3,2 \times 4$, etc.) are then placed into kiln dryers according to the loading pattern corresponding to that dimension for various duration in order to decrease their moisture content to an appropriate level (standard or required by the client). Once dried, bundles are disassembled to be sorted and graded according to standard rules or customers specifications, then cut to length and planed. The overall finished products are made of various grades and various sizes of pieces of lumber that are assembled into homogeneous bundles. These bundles are then sent to their final customers, which include wholesalers, retailers, and industrial customers for a second transformation into prefabricated houses or house components.

In this typical example, a single company is responsible for orchestrating all operations from the forest to the customers. Such a company may also orchestrates the operations of many sawmills while presenting a single face to customers through a corporate office responsible for managing customer relationships and sales. Sawmill operations complexity arises due to its divergent production process, the current push-mode production strategy of the industry and the heterogeneous nature of the resource. These specific industrial characteristics make it difficult to anticipate with precision the production output mix and volume.

\subsection{Application}

In the context of this application, many decisions must be addressed and coordinated. Here, we illustrate only part of the global decision process and its relationships with the platform. The architecture described earlier and the specific application presented hereafter have been implemented with Microsoft .NETC and Ilog OPL Studio(C) (which includes Cplex(C) and Solver(C) for the optimization part.

This application is dedicated to the planning of lumber production units. In those units, logs are transformed into lumber that is then dried and finished (i.e., planed). Next, finished lumber is either delivered to customers from an internal warehouse, or 
to an external warehouse. The design of the agent-based planning system for such a specific application is guided by the straightforward identification of homogeneous planning domains that can be found in the management of such supply chains: the planning of sawing, drying, finishing and transportation operations and the management of an external warehouse. Figure 3 illustrates the proposed design. In brief, sawmill planning unit is composed of generic agents: a planning unit manager agent (omitted in the figure), a deliver agent and a source agent. Then, specialized agents are introduced. A sawing agent, a drying agent and a finishing agent are respectively responsible for supporting the planning of sawing, drying and finishing operations. This specific internal structure is neither unique nor optimal. Although it is highly distributed, it is possible to design a more centralized structure with a single make agent responsible for the planning of all production operations. However, due to the complexity and the specificities of those problems, it seems difficult to take advantage of a single generic planning algorithm, as it is likely that only aggregated information could be handled with such an agent.

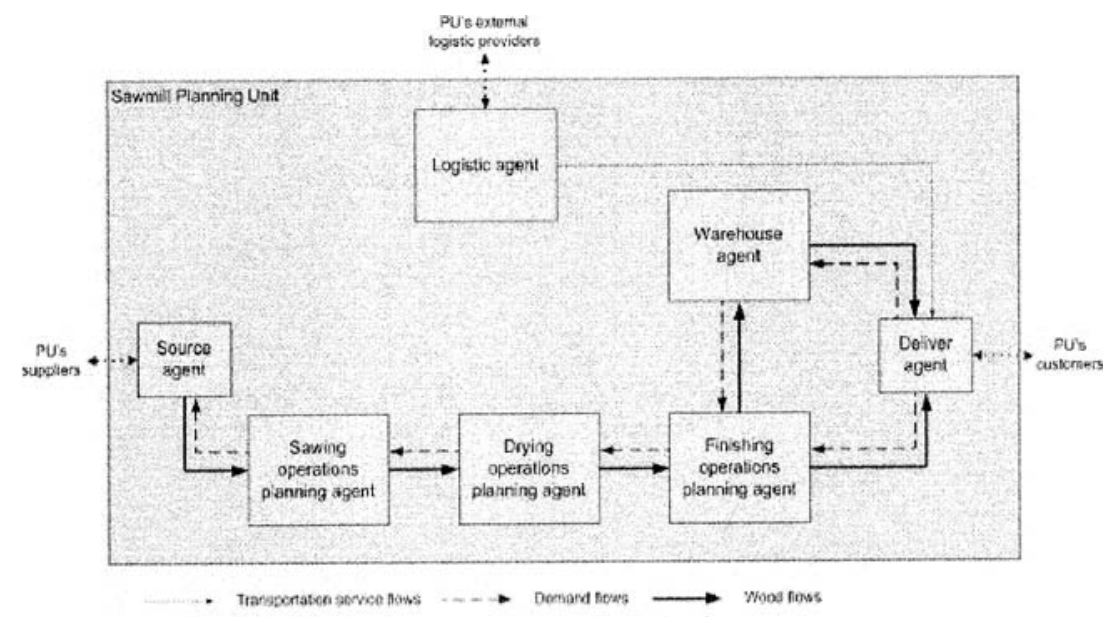

Figure 3 - Sawmill internal configuration

In brief, agents can calculate at any given time, a new requirement plan (i.e., a plan of its own needs to be sent to its supplier). The first requirement planning does not consider supply constraints and is referred to as the infinite sourcing capacity mode. The infinite sourcing capacity mode is meant to identify and communicate to the supplier the requirement plan that would best satisfy its local needs. In other word, it is seen as a target for the supplier that, in turn, trade-off the full satisfaction of its customer versus its own constraint and cost. Penalties for under or over satisfying the demand plan can be used in local planning models to allow such tradeoffs. Then, finite sourcing capacity mode can be used either after the receipt of a new replenishment plan from its supplier in order to provide its customer with an accurate plan to fulfill its needs, or using the current replenishment plan in order to provide its customer with a quick answer to a request. In this context, agents' behavior model must be designed in order to explicitly describe the context of using 
these specific configurations of their local planning models. In other words, these behavior models represent the agents' knowledge of how and when to use specific configurations of their planning tools. Research is currently done in order to address the explicit modeling of this kind of knowledge. It is however outside the scope of this paper.

\subsection{Validation}

The validation of these developments was carried out with the collaboration of a forest product company. Real data was thus used to test the performance of the agent-based APS. More specifically, we developed a configuration of the distributed APS in order to address the planning of drying and finishing activities inside one plant. This configuration included different types of data, such as production processes, products, orders, on-hand inventory, selling prices, resource costs, forecasted supply, capacity and on-going work. This test was covering 100 products, distributed on two dryers and one finishing line, in a planning horizon of 6 weeks.

The first step of this validation was to model the drying and finishing processes with the partner's production manager. Loading patterns for dryers were known and available, but finishing processes were unknown. Work has thus been done to define in details these processes, which resulted in 20 finishing processes and 89 drying processes. The customer's order file and the on-hand inventory data were extracted from their ERP system. The sales team provided the data on final product prices and resource costs. Each week, the partner's production manager sent us the execution plan, including supply from the sawing line, daily capacity of the finishing line and on-going work. The needed information were then translated in XML format and introduced into the experimental platform. Approximately 80 exchange protocols, 100 tasks and 50 workflows were involved in the experimental planning platform.

We then generated production and logistics plans and presented these to the production manager, for comments. This interactive validation phase has permitted to review and adjust the planning parameters and algorithms. Moreover, by studying the real plan prepared by the manager, we were able to evaluate the performance of the platform in terms of number of late customer orders, production value, resource utilization, etc. These indicators, easily obtained by the platform, were precious to evaluate the performance of both plans and identify possible improvements.

This validation process took about one year and many corrections have been made on the platform. Now, plans generated by the platform offer considerable improvements, while compared to plans prepared by the partner's production manager. Also, the actual planning times for drying and finishing operations have been reduced dramatically.

\section{CONCLUSION}

This paper has presented a software architecture which aims at designing distributed APS tools for the forest products industry. The presented platform follows the double objective of providing the industry with advanced planning tools, but also with a means of studying the dynamic and performance of such tools working together. The first objective has been already addressed, and quantitative testing of these tools in a large scale context (i.e., real industrial test case) has been 
undertaken. Concerning the second objective, a research effort has been initiated. This effort focuses on the simulation part of the platform. In other words, this effort aims at designing the mechanisms and software components required to put such distributed advanced planning tools in a simulated environment in order to test them in a live (though simulated) environment.

Finally, another research direction that is currently investigated concerns the agents' architecture. Agents in such a context seem to require the ability to exploit planning tools in various configurations according to the situation. In this context, it seems necessary to provide agents with sufficient knowledge and competencies about the planning tools and their environment, in order to deal timely with different situations by using the different available tools in the most appropriate way.

\section{ACKNOWLEDGMENTS}

This work was funded by the FOR@C Research Consortium and the Natural Sciences and Engineering Research Council of Canada (NSERC).

\section{REFERENCES}

1. Caridi M, Cavalieri S. Multi-agent systems in production planning and control: an overview. Prod Plan Control 2004; 15(2):106-118.

2. Dudek G, Stadtler G. Negotiation-based collaborative planning between supply chain partners. Eur J Oper Res 2005; 163(3): 668-687.

3. Epstein R, Morales R, Seron J, Weintraub A. Use of OR Systems in the Chilean Forest Industries. Interfaces 1999; 29(1): 7-29.

4. Fleischmann B, Meyr H. Planning Hierarchy, Modeling and Advanced Plaming Systems. In Handbooks in OR\&MS, Elsevier, 2003.

5. Fox MS, Barbuceanu M, Teigen R. Agent-Oriented Supply-Chain Management. Int J Flex Manuf Sys 2000; 12:165-188.

6. Frayret JM., D'Amours S, Montreuil B. Coordination and Control in Distributed and Agent-Based Manufacturing Systems. Prod Plan Control 2004; 15(1): 42-54.

7. Frayret JM, Boston K, D'Amours S, Lebel L. The E-nabled Supply Chain - Opportunities and Challenges for Forest Business. Working paper of the CENTOR, Université Laval, 2005.

8. Parunak HVD. Industrial and Practical Applications of DAI. in Weiss, G. (Ed.), Multiagent Systems a Modern Approach to Distributed Artificial Intelligence, Cambridge (MS): MIT Press, 1999.

9. Rönnqvist M. Optimization in forestry. Mathematical Programming, Series B 2003; 97(1-2): $267-$ 284.

10. Shen W, Norrie DH. Agent-Based Systems for Intelligent Manufacturing: A State-of-the-Art Survey. Knowl Inf Syst 1999; 1(2): 129-156.

11. Shen W, Norrie DH, Barthes JP. Multi-Agent Systems for Concurrent Intelligent Design and Manufacturing. London: Taylor \& Francis, 2001.

12. Stadtler H, Kilger C. Supply Chain Management and Advanced Planning - Concepts, Models, Software and Case Studies. Berlin: Springer, 2000.

13. Stadtler H. Supply chain management and advanced planning-basics, overview and challenges. Eur J Oper Res 2005; $163(3): 575-588$.

14. Tharumarajah A. Survey of resource allocation methods for distributed manufacturing systems. Prod Plan Control 2001; 12(1): 58-68.

15. Weiss G. Multiagent Systems A modern Approach to Distributed Artificial Intelligence. Cambridge (MS): MIT Press, 1999

16. Stephens S. The Supply Chain Council and the Supply Chain Operations Reference (SCOR) model: Integrating processes, performance measurements, technology and best practice. Logistics Spectrum $2000 ; 34(3): 16-18$ 